
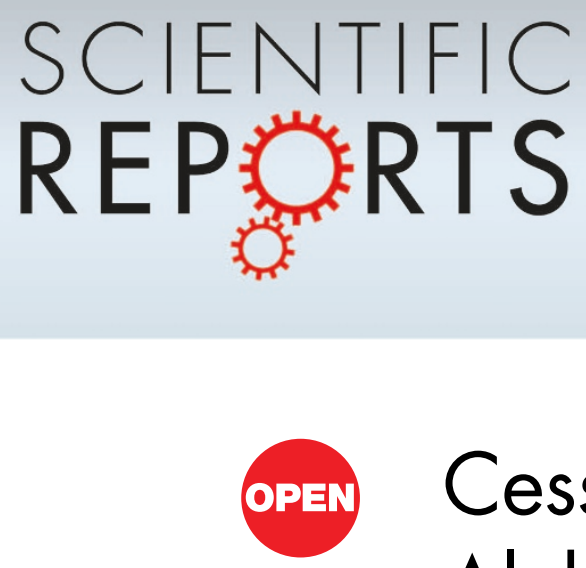

\title{
Cessation of Neoangiogenesis in Alzheimer's Disease Follows Amyloid-beta Immunization
}

SUBJECT AREAS: ALZHEIMER'S DISEASE

NEUROVASCULAR DISORDERS

BLOOD-BRAIN BARRIER

EXPERIMENTAL MODELS OF DISEASE

Received

5 October 2012

Accepted

25 January 2013

Published

28 February 2013

Correspondence and requests for materials should be addressed to W.A.J. (wilf@msl.ubc.

\author{
Kaan E. Biron 1,2,3,4,7, Dara L. Dickstein ${ }^{5}$, Rayshad Gopaul 1,2,3,4,6, Franz Fenninger 1,2,3,4 \\ \& Wilfred A. Jefferies ${ }^{1,2,3,4,6,7,8}$
}

\begin{abstract}
'Michael Smith Laboratories, The University of British Columbia, 301-2185 East Mall, Vancouver, British Columbia, V6T 1Z4, Canada, ${ }^{2}$ The Biomedical Research Centre, University of British Columbia, 2222 Health Sciences Mall, Vancouver, British Columbia, V6T 1Z3, Canada, ${ }^{3}$ The Brain Research Centre, University of British Columbia, 2211 Wesbrook Mall, Vancouver, B.C. V6T 2B5, Canada, ${ }^{4}$ Centre for Blood Research, University of British Columbia, 2350 Health Sciences Mall, Vancouver, BC, V6T 1Z3, Canada, ${ }^{5}$ Department of Neuroscience, Kastor Neurobiology of Aging Laboratories, Mount Sinai School of Medicine, 1 Gustave L. Levy Place, Box 1065, New York, NY, 10029, USA, ${ }^{6}$ Department of Zoology, University of British Columbia, $2370-6270$ University Blvd., Vancouver, BC, V6T 1Z4, Canada, ${ }^{7}$ Department of Microbiology and Immunology, University of British Columbia, 1365-2350 Health Sciences Mall, Vancouver, BC, V6T 1Z3, Canada, ${ }^{8}$ Department of Medical Genetics, 1364-2350 Health Sciences Mall, Vancouver, BC, V6T 1Z3, Canada.
\end{abstract}

Pathogenic neoangiogenesis in Alzheimer's disease (AD) is due to amyloid-beta $(A \beta)$ and results in blood-brain barrier (BBB) leakiness in AD. It likely occurs as a compensatory response to impaired cerebral blood flow and provides a strong link between brain vascularity and AD. A $\beta$ immunotherapy is an experimental treatment for $\mathrm{AD}$; however, unexpected negative vascular side effects seen in early human clinical trials demonstrate that our knowledge of $\mathrm{A} \beta$ and $\mathrm{AD}$ pathogenesis is incomplete. We demonstrate that immunization with $\mathrm{A} \beta$ peptides neutralizes the amyloid trigger leading to neoangiogenesis and reverses hypervascularity in Tg2576 AD mice. This process resolves plaque burden suggesting that neoangiogenesis is a key mechanism underlying plaque formation. A meta-analysis demonstrated that hypervascular reversion in vaccinated Alzheimer's patients. This appears to be the first example of vascular reversion following any therapeutic intervention and supports the conclusion that modulation of neoangiogenesis may repair damage in the $\mathrm{AD}$ brain. 


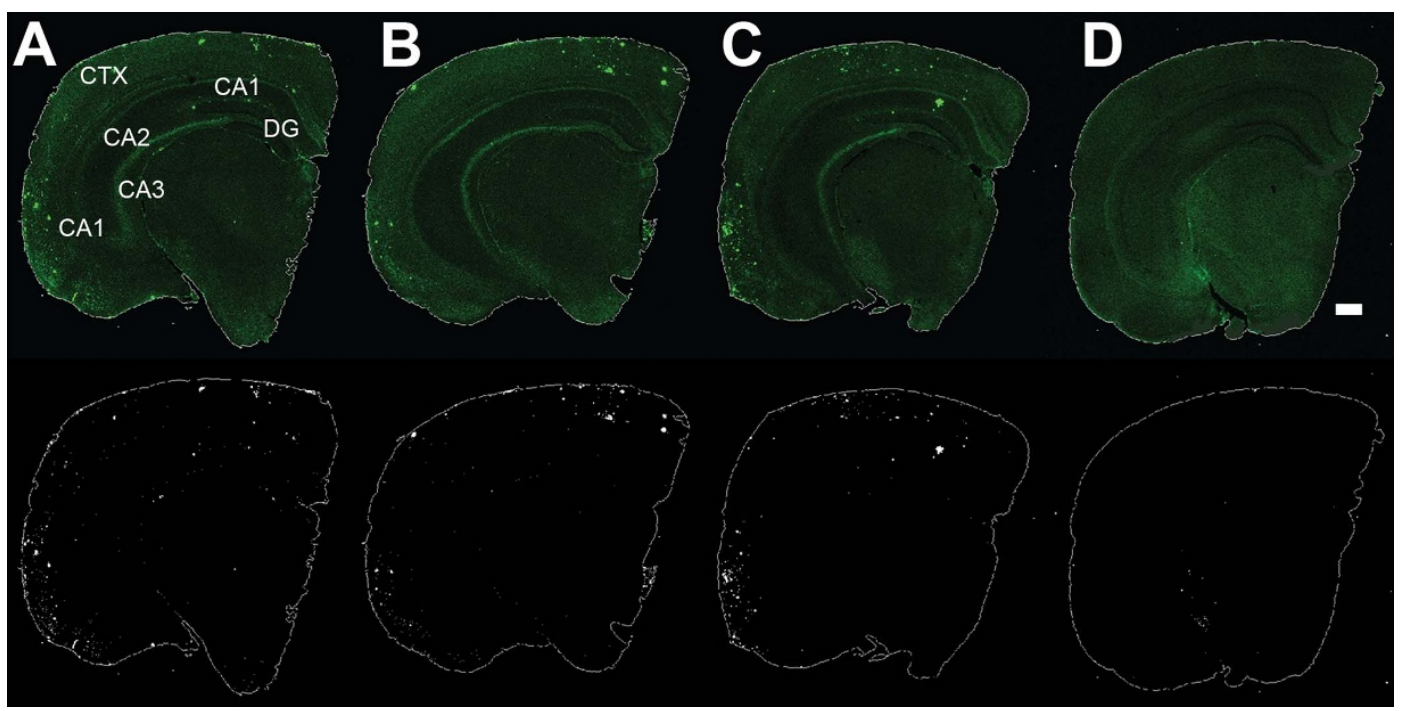

Figure 1 Reduced plaque pathology in Tg2576 mice immunized with A $\beta$. Immunized $\mathrm{Tg} /+$ mice, with either $\mathrm{A} \beta$ or PBS, were immunolabeled for $\mathrm{A} \beta$ plaques (green). Therapeutically immunized Tg/+ mice with PBS (A) or A $\beta$ (B). Preventatively immunized Tg/+ mice with PBS (C) or A $\beta$ (D). Lower panels correspond to the $A \beta$ threshold mask used to quantify the number of plaques, in white. Scale bar, $200 \mu \mathrm{m}$. The cortex (CTX) and hippocampus (CA1, CA2, CA3 and dentate gyrus (DG)) have been labelled. In all panels, data are representative of three experiments performed with A $\beta$ and PBS treated $\mathrm{Tg} /+$ mice as compared to the $+/+$.

The overall positive effects of the preclinical $A \beta$ immunization of $\mathrm{AD}$ mice encouraged a clinical human trial by Elan/Wyeth in late $1999^{11}$. However, unexpected negative vascular side effects ${ }^{12}$ prevented these therapies from reaching patients. The incomplete clinical trial had mixed results including reduced plaque pathology but persistent taupathies ${ }^{13}$. This demonstrates that our knowledge of $A \beta$, $\mathrm{AD}$ pathogenesis and the blood-brain barrier (BBB) is incomplete. Recently, we proposed the hypothesis that amyloidogenesis promotes extensive neoangiogenesis leading to increased vascular permeability and subsequent hypervascularization in $\mathrm{AD}^{5}$. We further hypothesized that active $\mathrm{A} \beta$ immunization might resolve this pathophysiological feature of $\mathrm{AD}$. Here we demonstrate that active immunization with $A \beta$ reverses $B B B$ breakdown at the level of the tight junction (TJ), which creates the physical seal of the BBB. Moreover we found that immunization with $A \beta$ caused a reversion of hypervascularization as indicated by a decrease in microvessel density to levels similar to control mice. While the exact mechanism for $A \beta$ immunotherapy remains to be clarified ${ }^{14}$, it is clear that the burden of $A \beta$ is reduced in the plasma and may directly or indirectly affect the vasculature. This appears to be the first example of vascular reversion following any therapeutic intervention and provides the proof of concept that neoangiogenesis modulation may repair damage in the AD brain.

\section{Results}

Immunized Tg2576 mice exhibit reduced A $\beta$ plaque pathology. $\operatorname{Tg} 2576(\mathrm{Tg} /+)$ mice immunized with $\mathrm{A} \beta$ had an overall reduction in parenchymal $A \beta$ plaques (Figure 1 ). Therapeutically treated $\mathrm{Tg} /+$ with $A \beta$ had a decrease in $A \beta$ plaque pathology (Figure 1B). A complete elimination in $A \beta$ plaques was seen in the $\mathrm{Tg} /+$ mice preventatively immunized with $A \beta$ (Figure 1D). The number of plaques was quantified (Figure 1 and Table 1), by image thesholding, confirming previous results ${ }^{10}$ showing an overall reduction plaque burden in $A \beta$ immunized $\operatorname{Tg} 2576$ mice.

Immunized Tg2576 mice exhibit reduced cerebrovascular tight junction pathology. $A \beta$ immunization can restore the $B B B$ integrity ${ }^{10}$ but the effect on the expression of TJ proteins like ZO-1 is unknown. Using both preventatively and therapeutically immunized mice, with either $\mathrm{A} \beta$ or PBS, the morphology of cerebrovascular
ZO-1 expression was imaged (Figure 2). Normal ZO-1 expression was visualized as strong, continuous and uninterrupted staining patterns. Indistinguishable regardless of brain region (neocortex or hippocampus), wild-type $(+/+)$ mice immunized with either $A \beta$ or PBS had normal ZO-1 staining patterns (Figure $2 \mathrm{~A}-\mathrm{D}$ ). $\mathrm{Tg} /+$ mice immunized both preventatively and therapeutically with $\mathrm{A} \beta$ exhibited normal ZO-1 expression in the capillaries (Figure 2G and $\mathrm{H})$ similar to $+/+$ mice. Abnormal expression appeared as weak, punctuate and/or discontinuous staining (white arrowheads; Figure 2E, F, I and J). Tg/+ mice receiving PBS had marked TJ pathology (Figure $2 \mathrm{E}$ and $\mathrm{F}$ ). Interestingly, Tg/ + mice immunized with $\mathrm{A} \beta$ displayed some abnormal TJ pathology mainly in larger vessels (Figure 2I and J). The shift in abnormal ZO-1 expression from the capillaries to the larger vessels in the $\mathrm{A} \beta$ immunized $\mathrm{Tg} /$ + mice mimicked the observed increase in vascular abnormalities observed in cerebral amyloid angiopathy (CAA) pathology ${ }^{12}$.

The incidence of TJ pathology was quantified by scoring the percentage of cerebrovasculature with intact versus abnormal ZO-1 morphology. Tg/+ mice immunized with PBS, both preventatively and therapeutically, had a significantly higher percentage of disrupted TJ expression in the neocortex and hippocampus compared to their $+/+$ counterparts immunized with PBS (Figure $2 \mathrm{~K}-\mathrm{N}$ ). Consistent with previous data ${ }^{10}, \mathrm{Tg} /+$ mice preventatively immunized with $A \beta$ displayed a significantly lower percentage of abnormal vascular TJ expression in the neocortex and hippocampus (averaging $10 \% ;{ }^{*} \mathrm{p}<0.05,2$-way ANOVA) compared to their PBS transgenic counterparts (Figure $2 \mathrm{~K}$ and $\mathrm{L}$ ). The level of TJ disruption in these mice was similar to $+/+$ controls injected with both A $\beta$ and PBS. Moreover, TJ pathology in $\mathrm{Tg} /+$ mice immunized therapeutically with $\mathrm{A} \beta$ exhibited a significant decrease (averaging $10 \%$; ${ }^{*} \mathrm{p}<0.05$,

Table 1 | A $\beta$ immunized Tg2576 mice have reduced plaque count. The number of $A \beta$ plaques and the percentage of $A \beta$ staining relative to the total area of the was quantified based on Figure 1

\begin{tabular}{lcccccc} 
& \multicolumn{2}{c}{ Therapeutically immunized } & & \multicolumn{2}{c}{ Preventatively immunized } \\
\cline { 2 - 3 } \cline { 5 - 6 } Immunogen & PBS & $\mathrm{A} \beta$ & & $\mathrm{PBS}$ & $\mathrm{A} \beta$ \\
\hline $\mathrm{A} \beta$ plaque count & 504 & 331 & & 199 & 55 \\
$\%$ of $\mathrm{A} \beta$ staining & 0.54 & 0.40 & & 0.38 & 0.04 \\
\hline
\end{tabular}



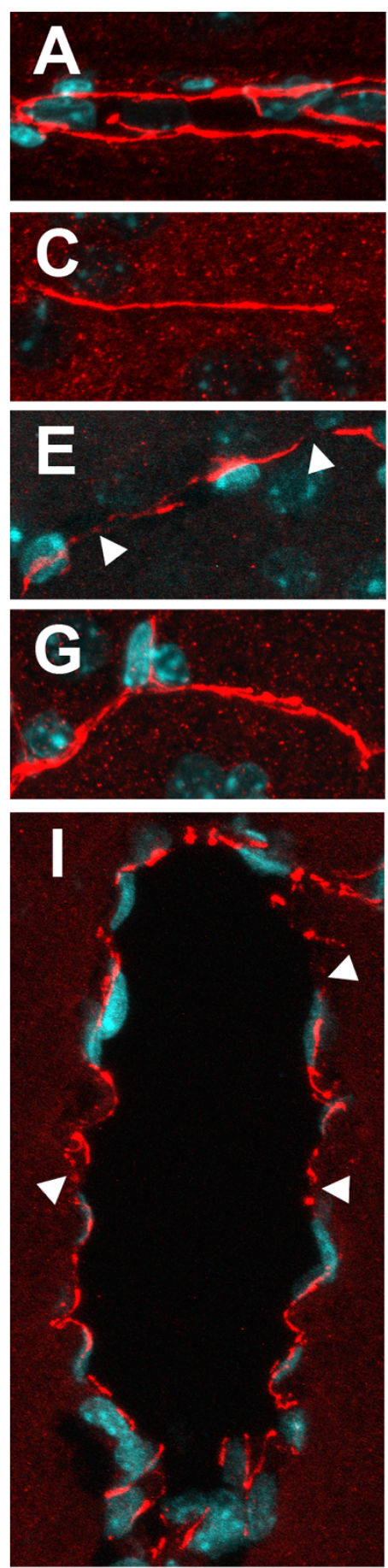

Preventative
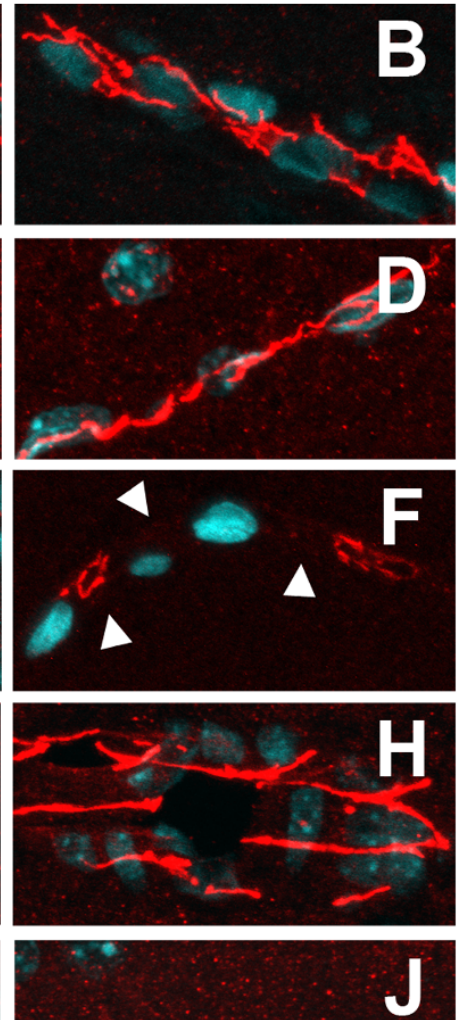

$\mathbf{J} \mathbf{M}$
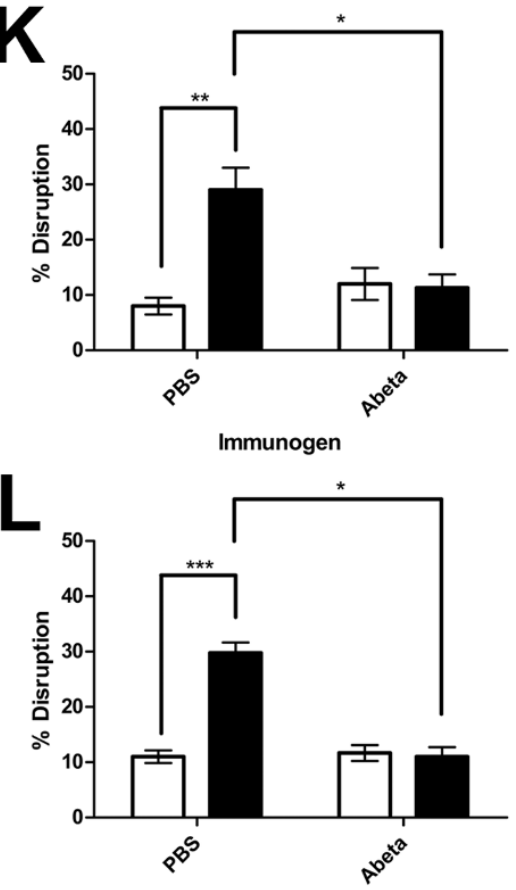

Immunogen

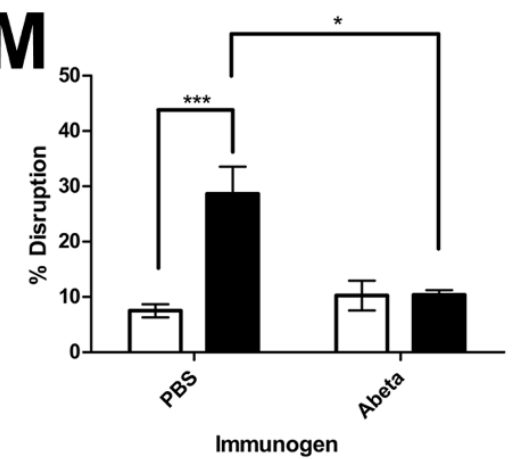

$\mathbf{N}$

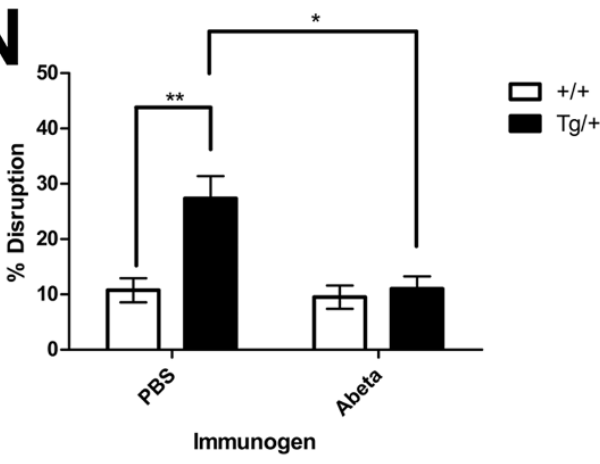

Figure 2 | A $\beta$ immunized Tg2576 mice had reduced ZO-1 abnormalities. Cerebrovasculature from $+/+(\mathrm{A}-\mathrm{D})$ and Tg/+ (E-J) mice immunolabeled for ZO-1 (red) and counterstained for DNA (blue) that were immunized with either with PBS (A, B, E and F) or A $\beta$ (C, D, G and H) preventatively or therapeutically. Normal ZO- 1 expression in $+/+$, PBS (A and B), $+/+, A \beta(C$ and D) and Tg/,$+ A \beta(G$ and $H)$. Abnormal ZO-1 expression in $\mathrm{Tg} /+$, PBS (E and F) and larger vessels of $\mathrm{Tg} /+, \mathrm{A} \beta$ (I and J). Scale bar, $20 \mu \mathrm{m}$. In all panels, micrographs are representative of three experiments performed with $\mathrm{A} \beta$ and $\mathrm{PBS}$ treated $\mathrm{Tg} /+$ mice as compared to the $+/+$. The percentage of cerebral blood vessels with abnormal ZO- 1 expression patterns was quantified. Results were consistent between immunization strategies, preventative $(\mathrm{K}$ and $\mathrm{L})$ and therapeutic $(\mathrm{M}$ and $\mathrm{N})$, and brain regions, cortex $(\mathrm{K}$ and $\mathrm{M})$ and hippocampus $(\mathrm{L}$ and $\mathrm{N})$. In the preventative strategy: $+/+, \mathrm{PBS}, \mathrm{n}=3 ; \mathrm{Tg} /+, \mathrm{PBS}, \mathrm{n}=4 ; \mathrm{A} \beta,+/+, \mathrm{n}=3 ; \mathrm{Tg} /+$, $\mathrm{A} \beta, \mathrm{n}=3$. In the therapeutic strategy: $+/+, \mathrm{PBS}, \mathrm{n}=4 ; \mathrm{Tg} /+, \mathrm{PBS} \mathrm{n}=3 ;+/+, \mathrm{A} \beta, \mathrm{n}=4 ; \mathrm{Tg} /+, \mathrm{A} \beta, \mathrm{n}=5$. Values are expressed as mean $\pm \mathrm{SEM}$ and are pooled from 3 separate experiments, ${ }^{*} \mathrm{p}<0.05,{ }^{*} \mathrm{p}<0.01,{ }^{* * *} \mathrm{p}<0.001$. 

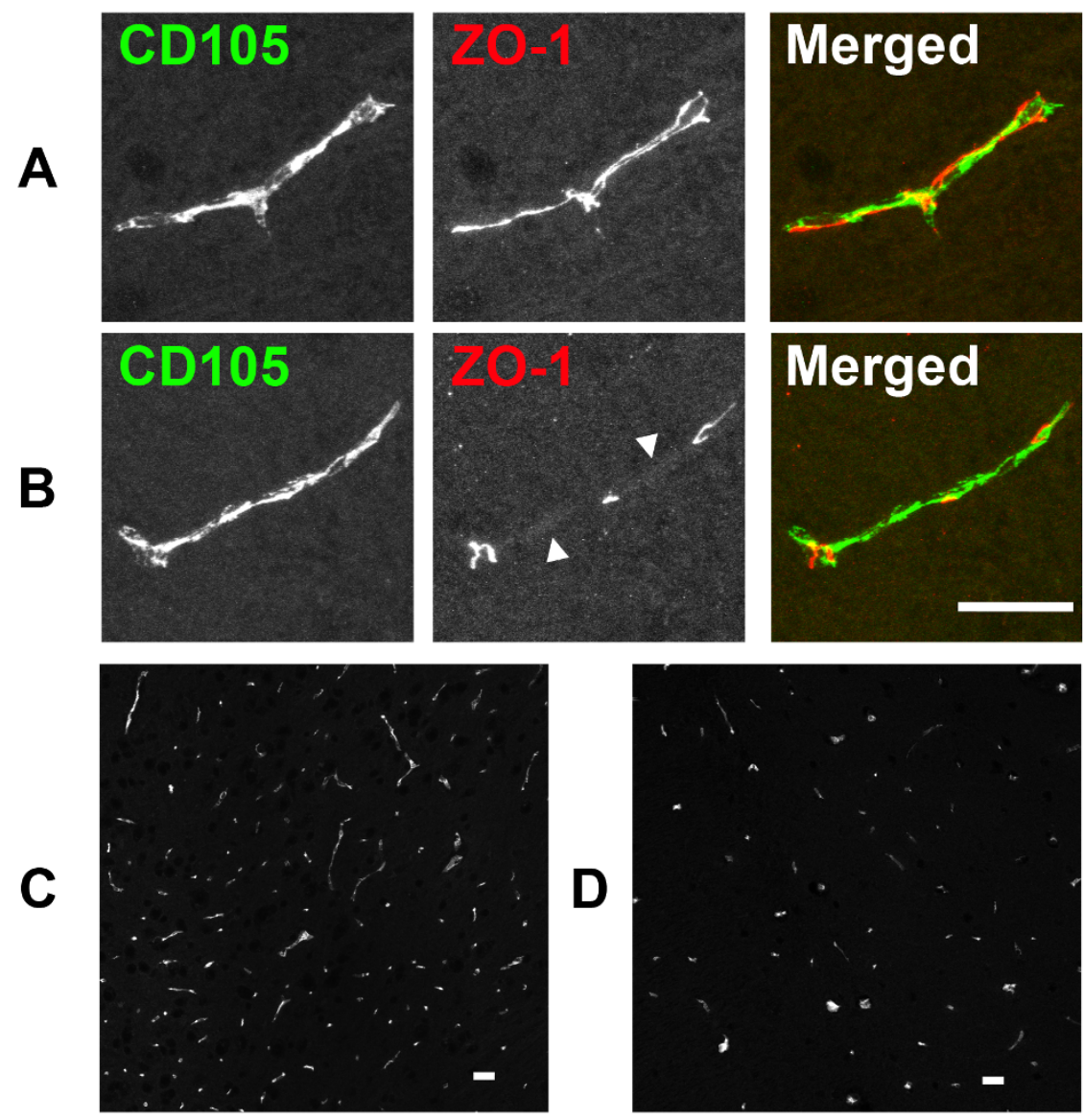

$\mathbf{E}$

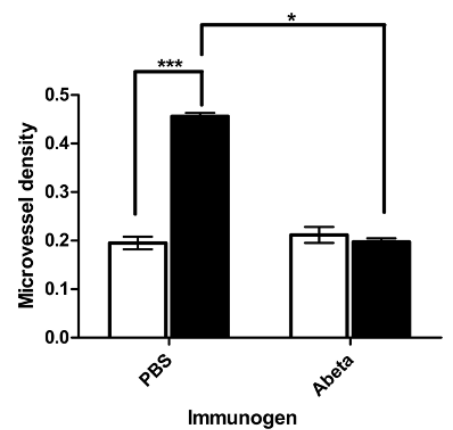

G

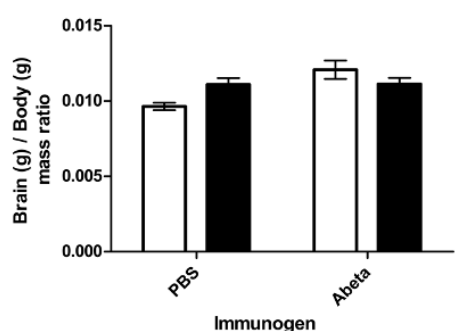

$\mathbf{F}$

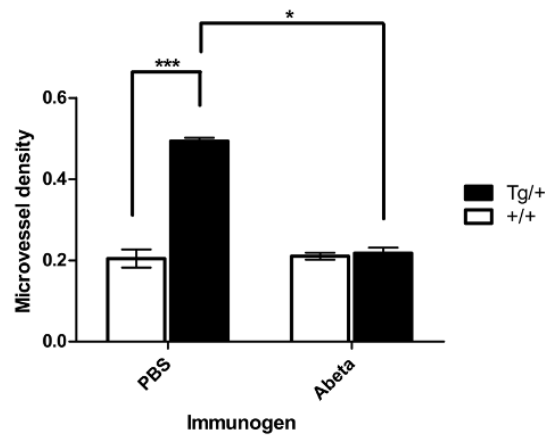

H
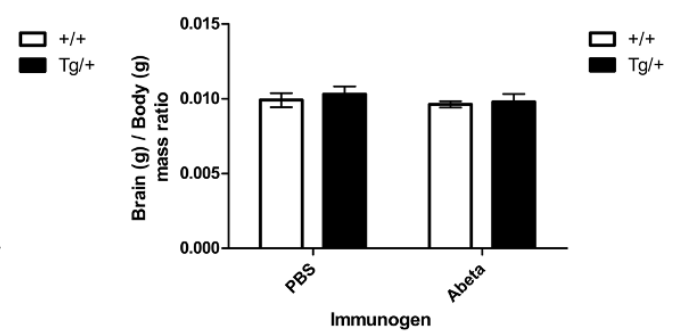

Figure 3 A A immunized Tg2576 mice have reduced microvascular density. CD105 (green) cerebrovasculature staining regardless of the (A) absence or (B) presence (white arrowheads) of ZO-1 (red) abnormalities. Ubiquitous "hotspot" staining of CD105 in the cortex of a preventatively immunized Tg/ + mouse with either (C) PBS or (D) A $\beta$. Scale bars, $20 \mu \mathrm{m}$. In all panels, micrographs are representative of three experiments performed with A $\beta$ and PBS treated $\mathrm{Tg} /+$ mice as compared to the $+/+$. Significantly MVD increased in Tg/+, PBS compared to Tg/+, A $\beta$ and $+/+, \mathrm{PBS}$ immunized

either (E) preventatively $(\mathrm{n}=4$ for all groups) and $(\mathrm{F})$ therapeutically $(+/+, \mathrm{PBS}, \mathrm{n}=4 ; \mathrm{Tg} /+, \mathrm{PBS}, \mathrm{n}=3 ;+/+, \mathrm{A} \beta, \mathrm{n}=4 ; \mathrm{Tg} /+, \mathrm{A} \beta, \mathrm{n}=4)$. The average brain to body weight ratio was not significant between mice immunized either $(\mathrm{G})$ preventatively $(+/+, \mathrm{PBS}, \mathrm{n}=5 ; \mathrm{Tg} /+, \mathrm{PBS}, \mathrm{n}=4$; $+/+, \mathrm{A} \beta, \mathrm{n}=5 ; \mathrm{Tg} /+, \mathrm{A} \beta, \mathrm{n}=3)$ or $(\mathrm{H})$ therapeutically $(+/+, \mathrm{PBS}, \mathrm{n}=4 ; \mathrm{Tg} /+, \mathrm{PBS}, \mathrm{n}=4 ;+/+, \mathrm{A} \beta, \mathrm{n}=5 ; \mathrm{Tg} /+, \mathrm{A} \beta, \mathrm{n}=5)$. Values are expressed as mean \pm SEM and are pooled from 3 separate experiments, ${ }^{*} \mathrm{p}<0.05, * * * \mathrm{p}<0.001$. 
2-way ANOVA) in TJ pathology compared to Tg/+ mice immunized with PBS in both the neocortex and hippocampus (Figure $2 \mathrm{M}$ and $N$ ). Furthermore, therapeutic $A \beta$ immunization was able to restore TJ protein expression to levels similar to $+/+$. It is important to note that PBS or $\mathrm{A} \beta$ treated $+/+$ mice resulted in minimal $\mathrm{TJ}$ disruption.

Angiogenesis in immunized $\mathrm{Tg} 2576$ brains. The microvascular density (MVD) is increased, by extension angiogenesis, in aged Tg2576 mice ${ }^{5}$ with disease progression and severity. Does active $\mathrm{A} \beta$ immunization alter the MVD in AD mice? CD105 staining was used to quantify the MVD, which was ubiquitous in the cerebrovasculature regardless of the absence (Figure 3A) or presence (Figure $3 \mathrm{~B}$ ) of TJ abnormalities. Representative examples of the ubiquitous CD105 "hotspot" staining in immunized (preventatively) Tg2576 mice with PBS (Figure 3C) or A $\beta$ (Figure 3D) demonstrates the MVD qualitatively. Tg/+ mice preventatively immunized with PBS had over double the MVD $(0.4560 \pm 0.0072$; $* * * \mathrm{p}<0.001,2$-way ANOVA) compared to their PBS immunized $+/+$ counterparts $(0.1951 \pm 0.0130)$ (Figure $3 \mathrm{E})$. In contrast, the MVD in Tg/ + mice preventatively immunized with $A \beta$ was significantly reduced compared to their PBS administered Tg/+ genotype counterparts $\left(0.1972 \pm 0.0075 ;{ }^{*} \mathrm{p}<0.05,2\right.$-way ANOVA) (Figure $3 \mathrm{E}$ ) and at a similar level observed in $+/+$ animals. The MVD in the therapeutically treated mice mirrored the observations in the mice immunized preventatively. $\mathrm{Tg} /+$ mice immunized after disease onset with PBS $(0.4939 \pm 0.0077)$ had over double the MVD compared to PBS-immunized $+/+$ mice $(0.2044 \pm 0.0222 ; * * * \mathrm{p}<$ $0.001,2$-way ANOVA) (Figure 3F). As seen in the $\mathrm{Tg} /+$ mice immunized prior to disease onset, $\mathrm{Tg} /+$ mice immunized with $\mathrm{A} \beta$ after disease onset also had a significantly reduced MVD $(0.2180 \pm$ $0.0130 ;{ }^{*} \mathrm{p}<0.05$, 2-way ANOVA) compared to Tg/+ mice immunized with PBS (Figure 3F). In short, Tg2576 mice immunized with A $\beta$ had significantly lower vascular densities (50\%) compared to wild-type mice and PBS treated Tg2576 mice. Changes in vascular density were independent of the physical size of the brains (Figure $3 \mathrm{G}$ and $\mathrm{H}$ ).

Furthermore, a Meta-analysis (see Appendix 1) that quantified the endothelial (laminin staining) in the grey and white matter in a vaccinated Alzheimer patient, as originally described by ${ }^{15}$, was conducted. Overall, brains from immunized patients had a reduced apparent vascular density compared to relevant controls. In the grey matter, 390 brain endothelia were identified in the untreated AD patient compared to 262 brain endothelia in the immunized $A D$ patient (a 33\% reduction after immunization). Similarly, in the white matter, 536 brain endothelia were identified in the untreated AD patient compared to 402 brain endothelia in the immunized $\mathrm{AD}$ patient (a $25 \%$ reduction after immunization).

\section{Discussion}

$A \beta$ immunotherapy continues to be explored as an experimental treatment option for AD. However, the unexpected negative vascular side effects seen in the early clinical trials of the human $\mathrm{AD}$ vaccine have prevented these therapies from reaching $\mathrm{AD}$ patients. The recent evidence suggests some optimism on this front ${ }^{16}$. Our objective in the current study was to determine if immunization with $A \beta$ peptides can resolve amyloidogenesis-triggered angiogenesis and hypervascularity in $\mathrm{Tg} 2576 \mathrm{AD}$ mouse. We find a dramatic reversion of hypervascularization follows immunization with $A \beta$. This appears to be the first example of vascular reversion where vascular density reverts to normal levels following therapeutic intervention. These findings clearly support a vascular angiogenesis model for $\mathrm{AD}$ pathophysiology and provide the first evidence that modulating angiogenesis, repairs damage in the $\mathrm{AD}$ brain.

$\mathrm{BBB}$ dysfunction was initially identified in animal models of $\mathrm{AD}^{17}$ and was later confirmed as a prominent, though unexplained, clinical feature of $\mathrm{AD}$ in patients ${ }^{18}$. We recently proposed a new hypothesis that is consistent with the literature relating to the $\mathrm{BBB}$ in $\mathrm{AD}$ : amyloidogenesis promotes extensive neoangiogenesis leading to increased vascular permeability and subsequent hypervascularization in $\mathrm{AD}^{5}$. In this model, pathological angiogenesis in $\mathrm{AD}$ may occur as a compensatory response to impaired $\mathrm{CBF}$ that induces neuroinflammation (releasing pro-angiogenic cytokines) and $A \beta$, which also acts as promoter of angiogenesis.

Here we demonstrate that $A \beta$ immunization modulates proangiogenic signals in treated Tg2576 mice that overexpress the human APP695 containing the double missense Swedish mutations (K670N/M671L), which causes early-onset AD. The relative amount of angiogenesis can be quantified through the average microvascular staining densities of CD105 in the brain ${ }^{19,20}$. The Tg2576 mice immunized with $A \beta$ had significantly lower vascular densities (50\%) compared to wild-type mice and PBS treated Tg2576 mice. This result was mirrored in a Meta-analysis in vaccinated human AD patients. Again this demonstrates that angiogenic signals are reduced when $A \beta$ is removed. It is presumed that neuroinflammation, a pathological feature of $\mathrm{AD}^{21}$, is reduced as a result of amyloid immunotherapy. The removal of the toxic $A \beta$ species $^{22}$ by active immunization would reduce neuroinflammatory cytokines like IL-1 $\beta$, which can induce angiogenesis ${ }^{23}$. While the direct involvement of $A \beta$ in influencing angiogenesis is controversial, recent evidence supports this hypothesis. Cameron et al. ${ }^{24}$ demonstrated $A \beta$ can directly influence angiogenesis via notch signalling. The authors suggest that $A \beta$ acts as competitive inhibitor to de-repress signals for angiogenesis.

In vivo (reviewed by ${ }^{9}$ ) and clinical ${ }^{25}$ studies have demonstrated the efficacy of active $A \beta_{1-42}$ immunotherapy in treating $\mathrm{AD}$ and $\mathrm{AD}$-like neuropathology; however, in this report we used $A \beta_{1-40}$ that replicated the earlier findings ${ }^{10}$. Although $A \beta_{1-40}$ and $A \beta_{1-42}$ have been cut from the same cloth, they do not share the same biochemical ${ }^{26}$ and pathophysiological properties. The soluble $A \beta_{1-40}$ species is associated with the $\mathrm{BBB}$ and therefore $\mathrm{CAA}^{27}$. The insoluble $\mathrm{A} \beta_{1-42}$ species is mainly found in senile plaques ${ }^{28}$. Post active vaccination with $A \beta_{1-42}$ results in the reduction of $A \beta_{1-42}$ (reduction in plaques) and evidence exists for the increase in $A \beta_{1-40}$ in patients ${ }^{29}$ (increase in CAA). Furthermore, an increase in cerebrovascular deposits of $\mathrm{A} \beta_{1-42}$ has also been observed ${ }^{30}$, which has been interpreted as part of a faulty perivascular drainage mechanism ${ }^{12}$.

A side effect observed in the failed clinical $A \beta$ immunization trial was increased CAA-associated cerebral microhemorrhaging ${ }^{31}$, also noted in a variety of $\mathrm{AD}$ mouse models after active ${ }^{32}$ and passive ${ }^{33}$ immunization. Normally, excessive $A \beta$ accumulation triggers neoangiogenesis resulting in the disruption of the TJs and BBB dysfunction. Microvascular leakage ensues allowing peripheral amyloid to enter the brain and coalesce as neurotoxic amyloid plaques. Cerebrovascular damage is further exasperated by and $A \beta$ induced ROS derived from NADPH-oxidase ${ }^{34}$. During immunization however, various $A \beta$ clearance mechanisms are activated including opsonization, microglia removal and antibody disaggregation. As $\mathrm{A} \beta$ plaques are dissolved, solubilized $A \beta$ is removed from the brain parenchyma along perivascular drainage routes ${ }^{35}$. Immunization therefore neutralizes the pro-angiogenic signal by stimulating an immune response to $\mathrm{A} \beta$ but other aspects of the disease, once formed, may be unresolved by immunization. For unknown reasons, the perivascular drainage of $\mathrm{A} \beta$ is halted and becomes deposited in the cerebral arteries, resulting in CAA. As noted earlier, the primary $A \beta$ species found deposited in the CAA-affected vasculature is the more soluble $A \beta_{1-40}$, believed to be of neuronal origin ${ }^{36}$. This model may ultimately explain the emergence of CAA in the failed human $\mathrm{AD} A \beta$ immunization trials ${ }^{31}$.

Recently, the anti-proliferative drug bexarotene (Targretin), an oral anti-cancer agent, has been shown to reduce plaque burden and increase memory performance in animal models of $\mathrm{AD}^{37}$. This study interprets bexarotene acting on retinoid $\mathrm{X}$ receptors to 
upregulate transcription of apoE, a protein that is implicated in amyloid beta plaque degradation ${ }^{37}$. The improvement in hippocampal function is ultimately attributed by the reduction of plaque accumulation or turnover, nevertheless, other modes of action in $\mathrm{AD}$ are possible. Bexarotene is also known to modulate the transcription of genes that are involved in cell migration, proliferation and apopto$\mathrm{sis}^{38}$. Moreover, in vitro, bexarotene has shown to directly exhibit an anti-angiogenic effect by downregulating pro-angiogenic factors ${ }^{39}$. These data and those presented herein, therefore directly point towards reversing cerebrovasculature angiogenesis as a new therapeutic modality for $\mathrm{AD}$.

\section{Methods}

Mice. Tg2576 transgenic $(\mathrm{Tg} /+)$ mice were used in this study. These mice express human APP695 containing the Swedish mutation (K670N/M671L $)^{40}$, under control of the hamster prion protein promoter (Taconic). Mice were maintained on mixed C57Bl6/SJL background by mating heterozygous Tg2576 males to C57Bl6/SJL F1 females. Wild-type $(+/+)$ littermates were used as controls. Mice were fed standard lab chow and water ad libitum and kept under a 12 hour light/dark cycle. All animal procedures were conducted with approval by the University of British Columbia Animal Care Committee.

A $\beta$ vaccination. $A \beta$ vaccination was performed as described by ${ }^{10}$. Briefly, two separate active vaccination strategies were carried out, therapeutic and preventative. In the preventative immunization approach, mice were vaccinated beginning at 6 weeks of age and sacrificed at 12 months of age. Mice used in the therapeutic strategy were vaccinated beginning at 11 months of age and sacrificed at 15 months of age. A $\beta$ peptide was freshly prepared from lyophilized powder for each set of injections. For immunizations, $2 \mathrm{mg}$ of $\mathrm{A} \beta$ (human $\mathrm{A} \beta_{1-40}$, Bachem) was added to $0.9 \mathrm{~mL}$ of deionized water and thoroughly mixed. Then $100 \mu \mathrm{L}$ of $10 \times$ PBS was added to obtain a final $1 \times$ PBS concentration. The solution was vortexed and placed at $37^{\circ} \mathrm{C}$ overnight until use the next day. $A \beta_{1-40}$ (100 $\mu$ g antigen per injection) or PBS (control) was mixed 1:1 (v/v) with complete Freund's adjuvant (CFA) for the first immunization. This was followed by a boost with $\mathrm{A} \beta_{1-40}(100 \mu \mathrm{g})$ or PBS mixed $1: 1$ (v/v) with incomplete Freund's adjuvant (ICFA) at two weeks and monthly thereafter. From the fifth immunization onward, straight PBS or A $\beta$ were injected. Injections were performed i.p.

Tissue preparation. Tissues were prepared as previously described by ${ }^{10}$. Mice were terminally anesthetized with ketamine/xylazine $(100 \mathrm{mg} / \mathrm{kg} ; 10 \mathrm{mg} / \mathrm{kg})$ and perfused with PBS for 5 minutes. Brains were then rapidly excised, olfactory bulbs and cerebellum removed, weighed and post-fixed in $4 \%$ paraformaldehyde for four days at $4{ }^{\circ} \mathrm{C}$. The brains were then imbedded in paraffin and sectioned serially at $5 \mu \mathrm{m}$. Paraffin embedding, sectioning, and dewaxing were performed by Wax-it Histology Services Inc. (Vancouver). Average brain to body mass ratios were compared amongst the preventatively and therapeutically mice.

Immunostaining. Dewaxed paraffin sections underwent antigen retrieval using a conventional stovetop pressure cooker using $20 \mathrm{mM}$ Tris with $0.7 \mathrm{mM}$ EDTA buffer ( $\mathrm{pH}$ 9.0) at full steam for 5 minutes. Cooled slides were then incubated in blocking buffer (25\% normal goat serum; 3\% BSA; 0.3\% Triton X-100, Sigma) for 1 hour at room temperature. Primary antibodies used included rabbit anti-ZO-1 $(1: 200$, Invitrogen), mouse anti-human CD105 ( $1: 20, \mathrm{DAKO})$, and mouse anti-A $\beta_{1-16}$ (6E10) (1:2000, Covance). Primary antibody staining was performed overnight at $4 \mathrm{C}$ in staining buffer ( $10 \%$ normal goat serum; $3 \%$ BSA; $0.3 \%$ Triton X-100). Secondary antibodies used were complimentary to the species of the primary conjugated with either Alexa Fluor dyes 488 or 568 (1:500, Invitrogen). Secondary antibody staining was performed at room temperature for 1 hour in staining buffer. TOTO-3 (1:10000, Invitrogen) was used for nuclear counterstaining. Sections were washed in PBS with $0.1 \%$ Tween-20 (Sigma) three times for 5 minutes each between staining steps. Stained sections were coverslipped using Fluoromount-G (Southern Biotech) and allowed to air dry in the dark overnight.

Confocal and quantitative analysis of tight junction morphology. Brain sections were analyzed from paraffin blocks from every fifth section. Images were taken on a Zeiss LSM510 Meta (Zeiss, Germany) using a $40 \times / 1.3$ oil-immersion Plan-Neoflaur objective. The composite projected image was imported into Adobe Photoshop, at $600 \mathrm{dpi}$, and optimized for contrast and brightness. Quantitative analysis of tight junction morphology was analyzed according the methodology described previously ${ }^{5}$. Confocal data sets represented approximately 100 cerebral blood vessels from both young and aged Tg2576 and littermate controls in the frontal cortex and hippocampus. Individual vessels were scored as either normal (1) or abnormal (0) for ZO-1 expression. Normal ZO-1 expression was judged as strong, continuous, intense and linear staining. In contrast, abnormal ZO-1 expression was judged as weak, punctate and/or discontinuous staining. Abnormal ZO-1 blood vessel expression was compared to normal blood vessels found in normal control or in normal vessels in diseased brains. To minimize the recording of incomplete or undulating vessels as abnormal, due to observed "gaps" in ZO-1 staining, evidence of vessel continuity was sought in the images. For example, the presence of stained nuclei (with TOTO-3) or punctate or diffuse ZO-1 remnants was used to localize the position of abnormal gaps along the vessel tract. The incidence of tight junction disruption was defined as the average percentage of blood vessels in a given region of brain that displayed abnormal tight junction morphology. A $\beta$ was imaged with a Zeiss LSM 710 Laser Scanning.

Microvessel density quantification. Microvessel density was quantified by confocal microscopy using the methods described previously ${ }^{5}$. Using CD105 as a brain angiogenic endothelial marker ${ }^{19}$, images of optimal fluorescent intensity were acquired and analyzed using the Zeiss LSM510 Meta software. Areas within the brain section containing high density ("hotspots") CD105 staining were imaged using the $20 \times / 0.45 \mathrm{~N}$-Achroplan objective using the confocal imaging parameters mentioned previously. The total fluorescence area (TFA) in $\mu \mathrm{m}^{2}$ was integrated above background, by the software, for each hotspot. The average TFA from four different hotspots per mouse was quantified. The TFA was used as a numerical representation of the total microvessels stained by the CD105 antibody. The microvascular density of the imaged field was expressed as a ratio of the TFA to the total area of the image.

Meta-analysis of vascular densities in human tissues and $\mathrm{A} \beta$ quantification was performed with a custom script using MatLab (v7.10.0.499, R2010a). The edges of the sections were detected using a segmentation algorithm to calculate the total area of each section. A threshold value was then calculated using the Sobel operator, which was tuned to $50 \%$ to obtain a binary mask for each section. To quantify $A \beta$ and laminin, the images were first converted into grayscale and then into black and white, using a threshold level of 0.6. Every pixel above this threshold level has been quantified as either $\mathrm{A} \beta$ or laminin, respectively. Eight or more connected white pixels, within the mask, were considered as either a plaque or endothelia. The percentage of $\mathrm{A} \beta$ staining was calculated by dividing the number of pixels above the threshold by the number of pixels of the total area of the tissue section. Images used in the Metaanalysis have been cropped to remove the original authors figure labelling. The Metaanalysis images were inverted to increase contrast.

Statistical analysis. All experiments were performed at least three times in triplicate. Statistical comparisons between $\mathrm{Tg} 2576(\mathrm{Tg} /+) \mathrm{AD}$ mice and wild-type $(+/+)$ control littermates immunized with PBS or A $\beta$ in the different immunization strategies were performed with 2-way ANOVA for unmatched values with Bonferroni post-tests. All statistical analyses were performed using GraphPad Prism (v5.01 for Windows, GraphPad Software, San Diego California USA, www.graphpad.com). $p$-values less than 0.05 were considered significant.

1. Castellani, R. J., Rolston, R. K. \& Smith, M. A. Alzheimer disease. Dis Mon 56, 484-546 (2010)

2. Hardy, J. Has the amyloid cascade hypothesis for Alzheimer's disease been proved? Curr Alzheimer Res 3, 71-73 (2006).

3. Zlokovic, B. V. Neurovascular pathways to neurodegeneration in Alzheimer's disease and other disorders. Nature reviews. Neuroscience 12, 723-738 (2011).

4. Paul, J., Strickland, S. \& Melchor, J. P. Fibrin deposition accelerates neurovascular damage and neuroinflammation in mouse models of Alzheimer's disease. J Exp Med 204, 1999-2008 (2007).

5. Biron, K. E., Dickstein, D. L., Gopaul, R. \& Jefferies, W. A. Amyloid triggers extensive cerebral angiogenesis causing blood brain barrier permeability and hypervascularity in Alzheimer's disease. PLoS ONE 6, e23789 (2011).

6. Neugroschl, J. \& Sano, M. Current treatment and recent clinical research in Alzheimer's disease. Mt Sinai J Med 77, 3-16 (2010).

7. Citron, M. Alzheimer's disease: strategies for disease modification. Nat Rev Drug Discov 9, 387-398 (2010).

8. Morgan, D. Immunotherapy for Alzheimer's disease. J Intern Med 269, 54-63 (2011).

9. Golde, T. E., Das, P. \& Levites, Y. Quantitative and mechanistic studies of Abeta immunotherapy. CNS Neurol Disord Drug Targets 8, 31-49 (2009).

10. Dickstein, D. L. et al. Abeta peptide immunization restores blood-brain barrier integrity in Alzheimer disease. FASEB J 20, 426-433 (2006).

11. Wilcock, D. M. \& Colton, C. A. Anti-amyloid-beta immunotherapy in Alzheimer's disease: relevance of transgenic mouse studies to clinical trials. J Alzheimers Dis 15, 555-569 (2008).

12. Vasilevko, V. et al. Aging and cerebrovascular dysfunction: contribution of hypertension, cerebral amyloid angiopathy, and immunotherapy. Annals of the New York Academy of Sciences 1207, 58-70 (2010).

13. Masliah, E. et al. Abeta vaccination effects on plaque pathology in the absence of encephalitis in Alzheimer disease. Neurology 64, 129-131 (2005).

14. Delrieu, J., Ousset, P. J., Caillaud, C. \& Vellas, B. 'Clinical trials in Alzheimer's disease': immunotherapy approaches. J Neurochem 120 Suppl 1, 186-193 (2012).

15. Boche, D. et al. Consequence of ACE $\leq$ immunization on the vasculature of human Alzheimer's disease brain. Brain 131, 3299-3310 (2008).

16. Menendez-Gonzalez, M., Perez-Pinera, P., Martinez-Rivera, M., Muniz, A. L. \& Vega, J. A. Immunotherapy for Alzheimer's disease: rational basis in ongoing clinical trials. Current pharmaceutical design 17, 508-520 (2011).

17. Ujiie, M., Dickstein, D., Carlow, D. \& Jefferies, W. A. Blood-brain barrier permeability precedes senile plaque formation in an Alzheimer disease model. Microcirculation 10, 463-470 (2003).

18. Farrall, A. J. \& Wardlaw, J. M. Blood-brain barrier: ageing and microvascular disease--systematic review and meta-analysis. Neurobiol Aging 30, 337-352 (2009). 
19. Holley, J. E., Newcombe, J., Whatmore, J. L. \& Gutowski, N. J. Increased blood vessel density and endothelial cell proliferation in multiple sclerosis cerebral white matter. Neurosci Lett 470, 65-70 (2010).

20. Barresi, V., Cerasoli, S., Vitarelli, E. \& Tuccari, G. Density of microvessels positive for CD105 (endoglin) is related to prognosis in meningiomas. Acta Neuropathol 114, 147-156 (2007)

21. Streit, W. J., Mrak, R. E. \& Griffin, W. S. Microglia and neuroinflammation: a pathological perspective. J Neuroinflammation 1, 14 (2004)

22. Benilova, I., Karran, E. \& De Strooper, B. The toxic Abeta oligomer and Alzheimer's disease: an emperor in need of clothes. Nat Neurosci 15, 349-357 (2012).

23. Pogue, A. I. \& Lukiw, W. J. Angiogenic signaling in Alzheimer's disease. Neuroreport 15, 1507-1510 (2004)

24. Cameron, D. J. et al. Alzheimer's-Related Peptide Amyloid-beta Plays a Conserved Role in Angiogenesis. PLoS One 7, e39598 (2012).

25. Nicoll, J. A. et al. Neuropathology of human Alzheimer disease after immunization with amyloid-beta peptide: a case report. Nat Med 9, 448-452 (2003).

26. Harrison, R. S., Sharpe, P. C., Singh, Y. \& Fairlie, D. P. Amyloid peptides and proteins in review. Rev Physiol Biochem Pharmacol 159, 1-77 (2007).

27. Suzuki, N. et al. High tissue content of soluble beta 1-40 is linked to cerebral amyloid angiopathy. Am J Pathol 145, 452-460 (1994).

28. Prelli, F., Castano, E., Glenner, G. G. \& Frangione, B. Differences between vascular and plaque core amyloid in Alzheimer's disease. J Neurochem 51, 648-651 (1988).

29. Patton, R. L. et al. Amyloid-beta peptide remnants in AN-1792-immunized Alzheimer's disease patients: a biochemical analysis. Am J Pathol 169, 1048-1063 (2006)

30. Nicoll, J. A. et al. Abeta species removal after abeta42 immunization. I Neuropathol Exp Neurol 65, 1040-1048 (2006).

31. Boche, D. et al. Consequence of Abeta immunization on the vasculature of human Alzheimer's disease brain. Brain 131, 3299-3310 (2008).

32. Wilcock, D. M. et al. Amyloid reduction by amyloid-beta vaccination also reduces mouse tau pathology and protects from neuron loss in two mouse models of Alzheimer's disease. J Neurosci 29, 7957-7965 (2009).

33. Racke, M. M. et al. Exacerbation of cerebral amyloid angiopathy-associated microhemorrhage in amyloid precursor protein transgenic mice by immunotherapy is dependent on antibody recognition of deposited forms of amyloid beta. J Neurosci 25, 629-636 (2005).

34. Park, L. et al. NADPH-oxidase-derived reactive oxygen species mediate the cerebrovascular dysfunction induced by the amyloid beta peptide. J Neurosci $\mathbf{2 5}$, 1769-1777 (2005).
35. Weller, R. O., Preston, S. D., Subash, M. \& Carare, R. O. Cerebral amyloid angiopathy in the aetiology and immunotherapy of Alzheimer disease. Alzheimers Res Ther 1, 6 (2009).

36. Herzig, M. C., Van Nostrand, W. E. \& Jucker, M. Mechanism of cerebral betaamyloid angiopathy: murine and cellular models. Brain Pathol 16, 40-54 (2006)

37. Cramer, P. E. et al. ApoE-directed therapeutics rapidly clear beta-amyloid and reverse deficits in AD mouse models. Science 335, 1503-1506 (2012).

38. Qu, L. \& Tang, X. Bexarotene: a promising anticancer agent. Cancer Chemother Pharmacol 65, 201-205 (2010).

39. Yen, W. C., Prudente, R. Y., Corpuz, M. R., Negro-Vilar, A. \& Lamph, W. W. A selective retinoid X receptor agonist bexarotene (LGD1069, targretin) inhibits angiogenesis and metastasis in solid tumours. British journal of cancer $\mathbf{9 4}$, 654-660 (2006).

40. Hsiao, K. et al. Correlative memory deficits, Abeta elevation, and amyloid plaques in transgenic mice. Science 274, 99-102 (1996).

\section{Acknowledgements}

The authors thank: Dr. Cheryl Pfeifer and Ms. Lonna Munro for critical reading and editing the manuscript; Kevin Hodgson of the UBC BioImaging Facility for technical advice; the Canadian Institute for Health Research for funding.

\section{Author contributions}

Conceived and designed the experiments: K.E.B., D.L.D., W.A.J. Performed the experiments: K.E.B., D.L.D. Analyzed the data: K.E.B., W.A.J. Contributed reagents/ materials/analysis tools: R.G., F.F. Wrote the paper: K.E.B., D.L.D., W.A.J.

\section{Additional information}

Supplementary information accompanies this paper at http://www.nature.com/ scientificreports

Competing financial interests: The authors declare no competing financial interests. License: This work is licensed under a Creative Commons Attribution-NonCommercial-NoDerivs 3.0 Unported License. To view a copy of this license, visit http://creativecommons.org/licenses/by-nc-nd/3.0/

How to cite this article: Biron, K.E., Dickstein, D.L., Gopaul, R., Fenninger, F. \& Jefferies, W.A. Cessation of Neoangiogenesis in Alzheimer's Disease Follows Amyloid-beta Immunization. Sci. Rep. 3, 1354; DOI:10.1038/srep01354 (2013). 